\title{
URBAN BUS FLEET ROUTING IN TRANSPORTATION NETWORK EQUIPPED WITH PARK-AND-RIDE: A CASE STUDY OF BABOL, IRAN
}

\author{
Amir Khakbaz ${ }^{1}$, Ali Shahandeh Nookabadi ${ }^{2}$, Seyyed Nader Shetab Boushehri ${ }^{3}$ \\ ${ }^{1,2}$ Dept of Industrial and Systems Engineering, Isfahan University of Technology, Iran \\ ${ }^{3}$ Dept of Transportation Engineering, Isfahan University of Technology, Iran
}

Submitted 28 January 2015; resubmitted 22 May 2015, 30 July 2015; accepted 12 August 2015

\begin{abstract}
Recently, most cities have tried to connect park-and-ride facilities with public transit vehicles. The present study aims to design urban bus routes in the transportation network equipped with park-and-ride. Seven important factors which affect the design of urban bus network are identified through the literature review. These factors include demand coverage, route directness, passengers' satisfaction, minimum length of bus route, budget, use of existing bus routes and number of lines. In this article, by use of the mentioned factors, a new model is developed to determine the urban bus routes. The new model figures the routes with park-and-ride as origin and Central Business District (CBD) as destination, in such a manner that the covered demand is maximized. Our novel method is more effective than other options currently available. In fact, it uses the most important factors in designing urban bus routes. Furthermore, an efficient Genetic Algorithm (GA) based approach is represented to solve large-scale problems. Numerical results show the effectiveness of this approach. At last, the developed model is applied to design the urban bus routes in the transportation network of Babol (Iran).
\end{abstract}

Keywords: urban transportation network design problem; urban bus routing; vehicle routing problem; park-and-ride; genetic algorithm.

\section{Introduction}

The citizens of most cities often face the problems of traffic congestion. Heavy traffic causes many problems, such as irregularity in the movement of vehicles, delay in performing everyday tasks, reducing the efficiency of citizens' tasks, noises, negative environmental impacts and increased stress caused by driving in high traffic density. Along with the development of cities, the growth in the number of private cars is inevitable and it causes traffic problems for city residents. In recent years, the expansion of public transit services and providing enough parking space for private cars have become the most important strategies to solve the traffic problems (Khoo et al. 2014). Using these strategies requires proper planning and management of urban transportation network.

Proper planning and management of urban transportation network is known as Urban Transportation Network Design Problem (UTNDP). Several aspects of these problems have been studied up to now, which two of them are mentioned in the following:

- designing new bus routes in the existing urban transit network to optimize the decision makers' purposes (Pacheco et al. 2009);

- finding optimal location of facilities added to the existing transportation network or determination of the optimum capacity of the existing facilities (Friesz 1985).

UTNDPs contain complicated solution methods and are important from the practical viewpoint. Therefore, a lot of research has been done and many books and articles have been published in this area in the past five decades. UTNDPs are divided into two categories (Farahani et al. 2013): Road Network Design Problem (RNDP) and Public Transit Network Design Problem (PTNDP). In RNDPs, the aim is to design new roads or develop the capacity of existed ones (Dantzig et al. 1979). On the other hand, PTNDPs are applied for designing public transit routes including origins, destinations and paths of incoming arcs by considering the existing network topology. Since in real world situations we rarely encounter the design of a new city, it can be said generally that PTNDPs are more applicable than RNDPs (Farahani et al. 2013). 
Public transit vehicles are used for high percentage of passengers' transportation in medium and large cities. Among these vehicles, urban buses are responsible for high proportion of passengers' transportation because of their cheapness and easy availability. The design of urban bus routes has direct impact on the travel time, passenger attraction and the total cost of public transit system. Hence, it is quite reasonable to design urban bus routes in such a manner that the travel time becomes as low as possible and the covered area becomes as high as possible. In addition, budget factor also must be considered in designing urban bus routes.

Nowadays, beside the proper design of public transit network and urban bus routes, it is tried to use parkand-ride facilities to improve the performance of public transit system (Khakbaz et al. 2013). Park-and-ride facilities are usually located on the periphery of the Central Business Districts (CBDs), and they prevent private cars from entering the CBD area. Equipping public transit system with park-and-rides and providing bus-based public transit services in park-and-rides can lead to reduced traffic congestion, higher satisfaction of private car users and extended use of public transit vehicles.

The performance of the park-and-rides can be strongly affected by the routes designed for urban buses which in turn depend on the factors which are used in designing the routes. The aim of the present study is to develop a mathematical model for finding optimal routes of urban buses in transportation network equipped with park-and-ride. In designing the urban bus routes, it is assumed that the urban buses start their trip form a park-and-ride and transport hundreds of passengers on their way to the CBD. To the best of our knowledge, there is no model that can simultaneously consider all mentioned factors. The proposed model finds the optimal routes in such a way that the all factors are considered and the covered demand is maximized.

In the section 1 of the article, public transit network is introduced briefly and previously developed models for designing public transportation network are reviewed. The basic assumptions of the new model are described in section 2, then some additional sets, parameters, and variables are defined and used to formulate the mathematical model. A GA-based algorithm for solving large-scale problems, is proposed in section 3 .
In section 4, a case study is presented to demonstrate the efficiency of the developed model and finally, the conclusion of this article is stated in the last section.

\section{Literature Review}

The urban transportation network design decisions are divisible into three levels: strategic, tactical and operational (Farahani et al. 2013). Decisions at each level are affected by those at the higher levels (Fig. 1). For example, making decisions about traffic light-timing largely depends on how to design and build the streets.

In recent years, many researches have been done on the design of urban transportation network that first of them was published in 1967. In the mentioned research, Lampkin and Saalmans (1967) developed a linear model for routing urban buses that aims to maximize the level of service they provide and then a heuristic approach was applied for solving the problem. From literature review, it is evident that effective factors in designing urban buses network are categorized into seven groups: demand coverage, route directness, passengers' satisfaction, minimum bus route length, budget, use of existing bus routes and number of lines. In the following, each of these groups is described separately.

Demand coverage. Bus-based transit services have the largest proportion of passenger transportation among different public transit modes. One of the most important characteristics of an urban bus fleet is its locality, meaning that the services provided by this fleet are available in most areas (Karou, Hull 2014). In other words, unlike rail-based transit services, such as metro and tram which are available just on rail lines, bus stops are available in many streets of a city. In addition to improving the use of bus-based transit services, proper design of urban bus routes also reduces the travel time and provides more satisfaction for passengers (Chakroborty, Wivedi 2002). Due to these facts, demand coverage maximization is one of the important goals in designing a public transit system (Ge et al. 2015). The area covered by a specific public transit system is equivalent to the portion of total transportation demand that would be met by the system. In many studies in the field of public transit network design, demand coverage has been regarded as an important criterion for measuring the efficiency of the final design. In some studies, the cov-

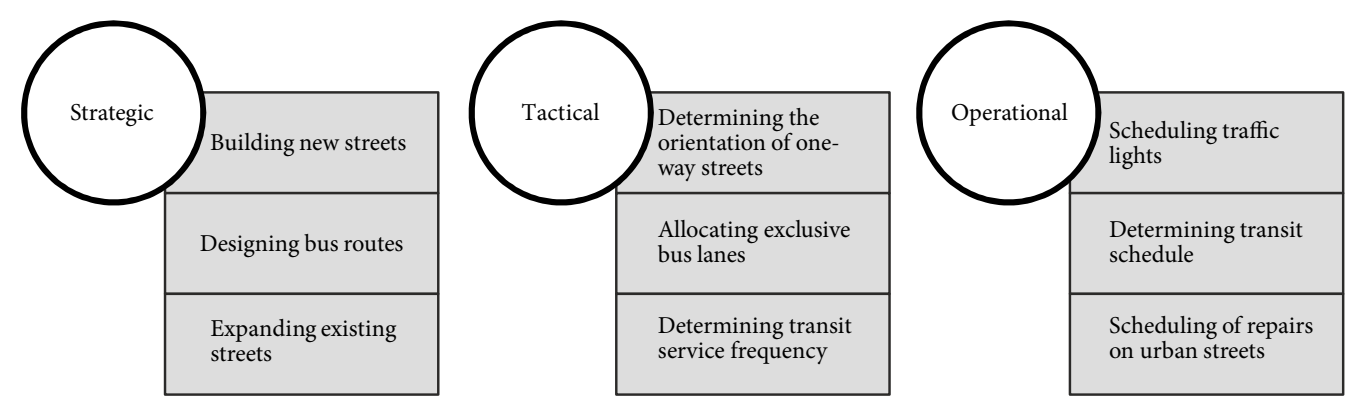

Fig. 1. Levels of decision-making process in the UTNDPs 
ered demand has been defined as an objective function to be maximized (Zhao 2006; Curtin, Biba 2011) and in some other studies, it has been considered as a constraint of the design problem (Barra et al. 2007; Nikolić, Teodorović 2013).

Route directness. Route directness is one of the criteria for evaluating the efficiency of urban bus transit that will be increased by decreasing bus route deviation from the shortest path. Directness of urban bus routes affects the speed of bus fleet, decreases travel time, and therefore it leads to a higher passengers' satisfaction. In some studies, route directness factor has been utilized for designing urban bus routes (Zhao, Zeng 2006; Zhao 2006).

Passengers' satisfaction. One of the main objectives in designing any public transit system is to increase passengers' satisfaction. So far, many definitions have been advanced for the term passengers' satisfaction but it can be said generally that passengers' judgments about the level of provided service reflect their satisfaction. If they were satisfied with the provided service, passengers would be more willing to use the service. The satisfaction level of urban bus passengers depend on several factors, including travel time, safety, information, convenience, driver behaviour and some others. Among them, travel time is one of the most important factors in designing urban bus routes (Thompson, Schofield 2007).

Minimum length of bus route. Determining the proper length of urban bus routes has a great impact on the performance of public transit systems. The shorter bus route will lead to a decrease in the cost of capital and the longer one will provide a more accessible service for passengers. Due to the impact of bus route length on the cost of capital and accessibility, some researchers have used this criterion in designing urban bus routes (Cipriani et al. 2012; Amiripour et al. 2014).

Budget. Along with the increase in the length of urban bus routes, there is an increase in the amount of fuel consumption, depreciation of equipment and labour requirements. In some studies, it has been tried to determine the optimal routes of urban buses by minimizing the total cost (Tirachini 2014; Badia et al. 2014) and in some other, budget has been considered as a constraint of the developed model (Yan et al. 2010; Tirachini et al. 2014).

Use of existing bus routes. The role of existing urban bus routes in designing new urban bus routes is discussable form two points of view including cost and passengers' satisfaction. Efficient use of existing urban bus routes reduces the cost of developing new routes, also, it increases the satisfaction level of passengers by keeping existing urban bus routes accessible (Bielli et al. 2002).

Number of lines. An increase in the number of urban bus lines will lead to an improvement in the level of provided service and make less traffic congestion. On the other side, this increment needs more investment on the public transit system that, due to budget constraint, can be reached partially. In several studies, it has been tried to determine the optimal number of lines by considering the length of bus routes and transport network topology (Fan, Mumford 2010; Szeto, Wu 2011).

Through literature review, it can be found that previous studies on the urban bus network design problems contain wide variety of objective functions and constraints, also, they have used one or more factors for modelling. Budget and minimum length of bus route factors have been used in several studies, which indicates the importance of them. However, all seven factors have not been used simultaneously in any of the studies reviewed. According to the importance of each factor in designing urban bus routes, it feels the need to develop a new model that incorporates the all important factors in designing urban bus routes. The Vehicle Routing Problem (VRP) is one of the most studied models which has attracted much interest in recent years and plays an important role in the field of distribution and transportation network design problems. In this research, a new VRP model is proposed that efficiently considers the all seven factors in designing urban bus routes.

Represented model is different from previous studies in the following key ways. First, objective function and constraints of the developed model are different from existing studies. Second, determining urban bus routes which originate from a park-and-ride and finish at $\mathrm{CBD}$ area is investigated for the first time. Demand coverage is the objective function to be maximized. Also, several constraints, such as route directness, number of lines, budget, maximum use of existing urban bus routes in designing new routes and minimum length of each route are considered in the new model. After formulating the model, a Genetic Algorithm (GA) based approach is represented for solving large-scale problems.

\section{Model Formulation}

The VRP is one of the best-known combinatorial optimization problems in which optimal solution is determined in such a way that the designed routes totally cover the demand of the customers (Lin et al. 2010). The first study in this area that used the term 'vehicle routing', was presented by Golden et al. (1977).

Transport network, users, vehicles, depots, operational constraints and objective function are the most important components of the VRP. The transport network is represented by a graph $G=(N, A)$ where $N$ is a set of nodes and $A$ is a set of undirected arcs. Nodes indicate the depots and the location of users that referred to as demand node while the arcs represent the path between two nodes. Users or passengers are people who use urban buses, referred to as vehicle, to travel from one node to another. Urban buses are used to transport passengers on their route from a park-and-ride, referred to as depot, to a demand node on the CBD area. The developed linear model tries to design the urban bus routes in such a manner that the total covered demand is 
maximized. The constraints of the model are: number of lines, number of park-and-rides, available budget, minimum length of each bus route, maximum use of existing urban bus routes and route directness.

In the all previous VRPs, vehicles are used to meet the demand between each pair of demand node and depot. In the developed model, urban buses start their trip form a park-and-ride and transport passengers on their route to a demand node on the CBD. Ultimately, they return to the park-and-ride by going through the route in the reverse order. In represented model, two types of demand are taken into account:

- the demand between each pair of demand node and park-and-ride: this type of demand refers to the passengers leaving their private vehicles at the park-and-ride and using urban buses for the rest of their trip to the CBD area; in other words, these passengers use private vehicle to travel from their origin, somewhere out of the CBD area, to the park-and-ride and continue their trip to the CBD by use of urban buses at the park-and-ride.

- the demand between each pair of demand nodes: this type of demand includes passengers that select urban buses for doing their trip form one node to another one in the CBD area; in other words, private vehicles do not have any role in their trip and they only use urban buses for doing their trip.

The new model uses a modal choice model for transit modes, including private vehicles, taxis, urban buses and non-motorized mode. In this research, a Multinomial-Logit Model (MNL) is used to determine the demands related to the bus network which are considered as the input of the new model (Tay et al. 2011). The multinomial choice model expresses the probability of individual $m$ to choose transportation mode $r$ within the choice set $C_{m}$ by the following equation:

$$
P\left(r \mid C_{m}\right)=\frac{e^{V_{r, m}}}{\sum_{o \in C_{m}} e^{V_{o, m}}},
$$

where: $C_{m}$ are the transportation mode alternatives which include private vehicles, taxis, urban buses and non-motorized mode; $V_{r, m}$ is the utility function of the $r$-th transportation mode; $V_{o, m}$ is the utility function of the $o$-th transportation mode.

This function is defined as follows:

$$
V_{r, m}=\mu_{\text {cost }}\left(c_{r, m}\right)+\mu_{t i m e}\left(d_{r, m}\right)
$$

where: $c_{r, m}$ and $d_{r, m}$ represent the perceived cost and duration of a trip respectively; the parameters $\mu_{\text {cost }}$ and $\mu_{\text {time }}$ allow to balance these two costs.

According to the characteristics of all developed VRPs and based on the mentioned assumptions, three different types of VRP models are used to develop a new model. These models include, Pickup and Delivery Problem (PDP), Multi-Depot Vehicle Routing Problem (MDVRP) and Open Vehicle Routing Problem (OVRP).
Following assumptions are also considered in developing the new model:

- urban buses are the only type of vehicle that used in the model;

- the travel time is deterministic;

- in each node, some passengers get off the bus and some passengers get on the bus;

- each urban bus route starts from a park-and-ride and finishes in a demand node at the CBD.

To formulate the developed model, some notations are introduced in this article and the transit network is expanded to include one dummy node (i.e., node 0 ). The dummy node is used to show that each new urban bus route finishes at the CBD area. The notations and parameters of this study are given in the following.

Indices:

$i, j, p, t, k, s$ - indices of nodes;

$\rho$ - indices of new urban bus routes;

$l$ - indices of existing urban bus routes.

\section{Parameters:}

$n$ - total number of nodes,

$n=\left\{\begin{array}{l}0 \text { indicates the dummy node; } \\ \text { park }- \text { and }- \text { rides are shown by } 1, \ldots, P R ; \\ \text { demand nodes are represented by } P R+1, \ldots, n ;\end{array}\right.$

PR - number of park-and-rides;

NR - number of new urban bus routes that must be added to the transit network;

$D^{k s}$ - demand between origin $k$ and destination $s$;

$R_{p}$ - Number of new urban bus routes originate from $p$-th park-and-ride;

$M$ - an infinite number;

$c_{i j}$ - cost of arc $(i, j)$;

$d i s_{i j}$ - length of arc $(i, j)$;

$B$ - available budget;

$L B$ - minimum length of each new urban bus route;

$\mathrm{NE}$ - number of existing urban bus routes;

$N_{l}$ - number of nodes on the existing urban bus route l;

$\mathrm{O}_{l}$ - an ordered set of nodes on the existing urban bus route $l ; O_{l}=\left\{i_{1}^{l}, i_{2}^{l}, \ldots, i_{N_{l}}^{l}\right\}$;

$S P^{k s}$ - length of shortest path between origin $k$ and destination $s$;

$\theta$ - upper threshold for the deviation of new urban bus routes from their shortest path.

\section{Decision variables:}

$z^{k s, \rho}-1$ if nodes $k$ and $s$ are located on the new urban bus route $\rho$; equal 0 otherwise;

$x_{i j}^{\rho}-1$ if arc $(i, j)$ is located on the new urban bus route $\rho$; equal 0 otherwise;

$u^{k s}-1$ if nodes $k$ and $s$ are connected by at least one urban bus route (new or existing); equal 0 otherwise; 
$y$ - free variable;

$E_{l}^{\rho}-1$ if existing urban bus route $l$ is considered in designing new urban bus route $\rho$; equal 0 otherwise;

$O D^{k s, \rho} 1$ if node $k$ is the origin and node $s$ is the destina-

- tion of new urban bus route $\rho$; equal 0 otherwise.

Based on the above definitions, linear model for routing urban buses is formulated as follows:

$$
\max Z=\sum_{k=1}^{n} \sum_{s=1}^{n} u^{k s} D^{k s}
$$

subject to:

$$
\begin{aligned}
& \sum_{i=0}^{n} x_{i t}^{\rho}=\sum_{j=0}^{n} x_{t j}^{\rho}, \\
& \rho=1, \ldots, N R \text {, } \\
& t=P R+1, \ldots, n \text {; } \\
& \sum_{i=1}^{P R} \sum_{j=0}^{n} x_{i j}^{\rho}=1, \\
& \rho=1, \ldots, N R \text {; } \\
& \sum_{j=P R+1}^{n} \sum_{\rho=1}^{N R} x_{p j}^{\rho}=R_{p}, \\
& p=1, \ldots, P R \text {; } \\
& \sum_{j=0}^{n} \sum_{\rho=1}^{N R} x_{0 j}^{\rho}=0 ; \\
& \sum_{i=P R+1}^{n} x_{i 0}^{\rho}=1 \text {, } \\
& \rho=1, \ldots, N R \text {; } \\
& z^{k s, \rho} \leq \frac{1}{2}\left(\sum_{t=0}^{n} x_{k t}^{\rho}+\sum_{t=0}^{n} x_{s t}^{\rho}\right), \\
& k=0,1, \ldots, n \text {, } \\
& s=0,1, \ldots, n, \\
& \rho=1, \ldots, N R \text {; } \\
& \left(1-z^{k s, \rho}\right) \leq M\left(1-\frac{1}{2}\left(\sum_{t=0}^{n} x_{k t}^{\rho}+\sum_{t=0}^{n} x_{s t}^{\rho}\right)\right), \\
& k=0,1, \ldots, n, \\
& s=0,1, \ldots, n, \\
& \rho=1, \ldots, N R \text {; } \\
& u^{k s} \leq \sum_{\rho=1}^{N R} z^{k s, \rho}, \\
& k=0,1, \ldots, n \text {, } \\
& s=0,1, \ldots, n \text {; } \\
& u^{k s} \geq \frac{\sum_{\rho=1}^{N R}\left(1-z^{k s, \rho}\right)}{N R}, \\
& k=0,1, \ldots, n \text {, } \\
& s=0,1, \ldots, n ;
\end{aligned}
$$

$$
\begin{aligned}
& \sum_{i=0}^{n} \sum_{j=0}^{n} \sum_{\rho=1}^{N R} c_{i j} x_{i j}^{\rho} \leq B ; \\
& \sum_{i=0}^{n} \sum_{j=0}^{n} d i s_{i j} x_{i j}^{\rho} \geq L B \\
& \rho=1, \ldots, N R ; \\
& y(i)-y(j)+\left(n \sum_{\rho=1}^{N R} x_{i j}^{\rho}\right) \leq n-1, \\
& i=P R+1, \ldots, n, \\
& j=P R+1, \ldots, n ; \\
& N_{l}-1, x_{i l}^{\rho}, i_{h+1}^{l}=E_{l}^{\rho}\left(N_{l}-1\right), \\
& h=1 \\
& l=1, \ldots, N E, \\
& \rho=1, \ldots, N R ; \\
& N R \\
& \sum_{\rho=1}^{N} E_{l}^{\rho} \geq 1, \\
& l=1, \ldots, N E ; \\
& O D^{k s, \rho} \leq \frac{1}{2}\left(x_{s 0}^{\rho}+\sum_{j=0}^{n} x_{k j}^{\rho}-\sum_{i=0}^{n} x_{i k}^{\rho}\right), \\
& k=1, \ldots, P R, \\
& s=P R+1, \ldots, n, \\
& \rho=1, \ldots, N R ;
\end{aligned}
$$$$
\left(1-O D^{k s, \rho}\right) \leq M\left(1-\left(\frac{1}{2}\left(x_{s 0}^{\rho}+\sum_{j=0}^{n} x_{k j}^{\rho}-\sum_{i=0}^{n} x_{i k}^{\rho}\right)\right)\right),
$$$$
k=1, \ldots, P R \text {, }
$$$$
s=P R+1, \ldots, n,
$$$$
\rho=1, \ldots, N R \text {; }
$$

$\sum_{i=1}^{n} \sum_{j=1}^{n} d i s_{i j} x_{i j}^{\rho} \leq\left(O D^{k s, \rho} \theta S P^{k s}\right)+\left(M\left(1-O D^{k s, \rho}\right)\right)$,

$k=1, \ldots, P R$,

$s=P R+1, \ldots, n$,

$\rho=1, \ldots, N R$;

$x_{i j}^{\rho}, z^{k s, \rho}, u^{k s}, E_{l}^{\rho}, O D^{k s, \rho} \in\{0,1\}, y \in I R$,

$$
\begin{aligned}
& i=0,1, \ldots, n, \\
& j=0,1, \ldots, n, \\
& k=0,1, \ldots, P R, \\
& s=0,1, \ldots, n, \\
& \rho=1, \ldots, N R, \\
& l=1, \ldots, N E .
\end{aligned}
$$


In the represented model, the objective function (3) maximizes total covered demand. Constraints (4) ensure the continuity of routes i.e. when an urban bus route enters in a node it has to exit. Constraints (5) show that each new urban bus route should be started from a parkand-ride. Constraints (6) define the number of urban bus routes that must be originated from each park-and-ride. Constraint (7) implies that there is no arc from node 0 to other nodes. Based on constraints (8), all new urban bus routes must be ended at node 0 . Constraints (9) and (10) determine whether the nodes $k$ and $s$ are located at the new urban bus route $\rho$. Constraints (11) and (12) determine whether the nodes $k$ and $s$ are connected to each other. Budget restriction is expressed by constraint (13). Based on Constraints (14), the length of each new urban bus route should at least be equal to $L B$. Constraints (15) prohibit subtour solutions. Constraints (16) ensure that if existing urban bus route $l$ is considered in designing new urban bus route $\rho$, then the sequence of nodes in the route $l$ is ordered. Constraints (17) impose that each existing urban bus route must be used at least in one of new urban bus routes. Constraints (18) and (19) determine the start and end nodes of each new urban bus route. Constraints (20) express that the deviation of each new urban bus route from its shortest path should not exceed the upper threshold.

This new model was developed based on the mathematical formulations of PDP, MDVRP and OVRP. Constraints (4)-(6) are those of the MDVRP. Constraints (7) and (8) belong to the OVRP, first proposed by Sariklis and Powell (2000) and constraints (9) and (10) are related to the PDP, another variant of the VRP, which considers pickup and delivery transportation demands simultaneously. Represented model contains both integer and continuous variables thus, it can be referred to as Mixed-Integer Linear Programming (MILP). Assuming the lack of pickup/delivery and second type of demand, developed model reduces to OVRP, which is NP-hard (Brandão 2004). Hence, represented model is also NPhard and an efficient heuristic algorithm is needed to obtain good solutions for large-scale problems.

\section{Solution Method}

In this article, a GA-based approach is applied for solving the developed model in a reasonable amount of time. GA is one of the most popular evolution algorithms and is more common in the field of UTNDP because of its efficiency and speed to achieve good solutions (Farahani et al. 2013). The developed GA-based approach first generates a number of feasible initial solutions, by use of a heuristic approach, that each of them indicates a set of urban bus routes originate from a park-and-ride and finish at the CBD area. Then, some parents are selected by Roulette Wheel method and children are produced through mutation and crossover. For each pair of selected parents, crossover produces a pair of children. Hence, the number of produced children is equal to the number of selected parents. In addition, some other parents are selected and new children produced by use of mutation. During the production of new children, an Evaluation operator is used to test the feasibility of the children. This operator checks the feasibility of budget limit, deviation from the shortest path and minimum length of each urban bus route for all produced children. All feasible children that produced through crossover and mutation operators, are transferred to next generation for producing new children and it continues until stopping criterion is met.

\subsection{Representation Scheme and Initialization Method}

A feasible solution includes $N R$ routes that $R_{p}$ of them start from $p$-th park-and-ride $N R=R_{1}+R_{2}+\ldots+R_{P R}$ and finish at a demand node in the CBD area. Fig. 2 illustrates the solution representation scheme of the GA-based approach in which squares indicate park-andrides, circles represent demand nodes and arrows show the direction of bus travel. The GA starts by generating npop feasible initial solutions by use of a heuristic approach which is described as follows:

\section{- Repeat}

- For route $\rho=1$ to $N R$

Select an origin node for route $\rho(i=1)$

- Repeat

Randomly select a node $i+1$ that is not appeared on route $\rho$

Insert node $i+1$ to route $\rho$

- Until the minimum length of route $\rho$ is reached and its deviation from shortest path is met

- Next route

- Until budget restriction is satisfied.

\subsection{Fitness Evaluation and Selection Method}

Fitness function value is the same as the objective function of the model. For each solution $i$ from the initial population npop, first the value of fitness function $f_{i}$ is

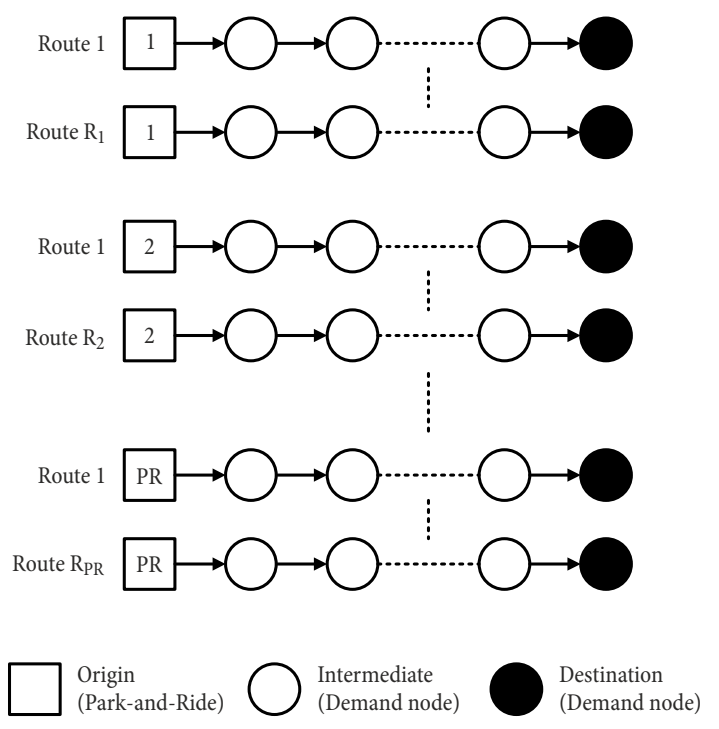

Fig. 2. Representation scheme of the GA-based approach 
calculated then selection probability of each individual $p_{i}$ is computed by Eq. (22). By use of Roulette Wheel method, a number of predefined random numbers are produced and corresponding individuals are selected for off-springs.

$$
\begin{aligned}
& p_{i}=\frac{f_{i}}{\sum_{j=1}^{n p o p} f_{i}} ; \\
& i=1,2, \ldots, \text {, } о \text { p } .
\end{aligned}
$$

\subsection{Crossover and Mutation Operators}

Because of the complexity of the represented problem in producing feasible solutions, specified crossover and mutation operators are used to develop the GA-based approach. Crossover operator exchanges sequences of intermediate nodes between two routes with the same origin and then the feasibility of produced children is investigated by Evaluation operator. In a sample problem with 2 park-and-rides and 18 demand nodes which is aimed to design one route from each park-and-ride, i.e. $R_{1}=R_{2}=1$, Fig. 3 illustrates the process of a crossover operator to produce new children.

Two mutation operators, namely, insert and remove are used for the developed GA (Fig. 4). In the insert operator, a stop node is inserted into a selected route of the solution which the node, the route and the inserted position are determined randomly. Likewise, the remove operator removes a node from a route randomly.
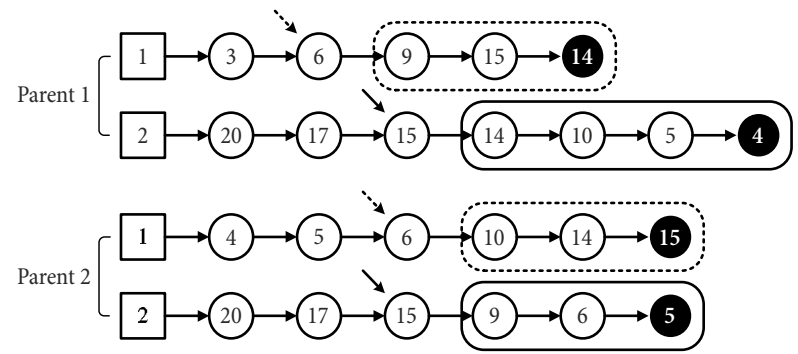

$\downarrow$
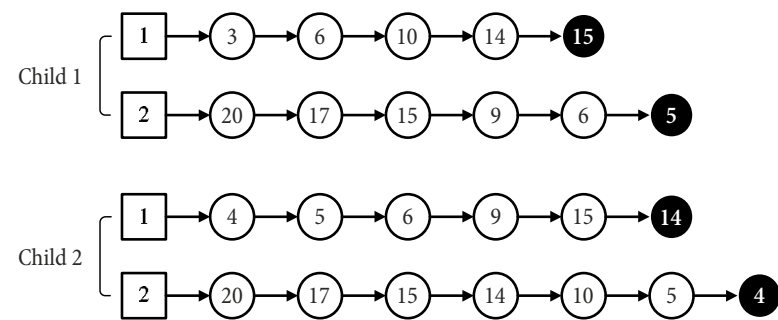

Fig. 3. Schematic illustration of crossover

\subsection{Stopping Criterion}

To improve the quality of the developed GAs solution, stopping criterion is defined based on the improvement in the results of successive generations. In other words, the algorithm stops when no improvement is observed for a predefined number of iterations.

\subsection{GAs Efficiency}

To evaluate the efficiency of the developed heuristic approach, 160 sample problems have been solved by use of the improved GA and the obtained results have been compared with the optimum solutions. For this comparison, the sample problems with an average optimum solution time less than 6000 seconds have been considered. These problems are divided into 8 major classes that each class exhibits the values of certain parameters of the model, such as the number of park-and-rides and the number of demand nodes. Other input data, such as demand matrix, distance matrix and cost matrix, were generated randomly for each of twenty problems belong to one of the eight main classes. The key parameters associated with the sample problems are summarized on Table 1.

Based on the results, in $92 \%$ of cases the solution obtained by the GA-based approach was the same as the optimal solution. The average time required to obtain the optimal solution was 5890 seconds that is so high in comparison with that of the GA-based approach, i.e. 94 seconds. By comparing optimal and the GA-based
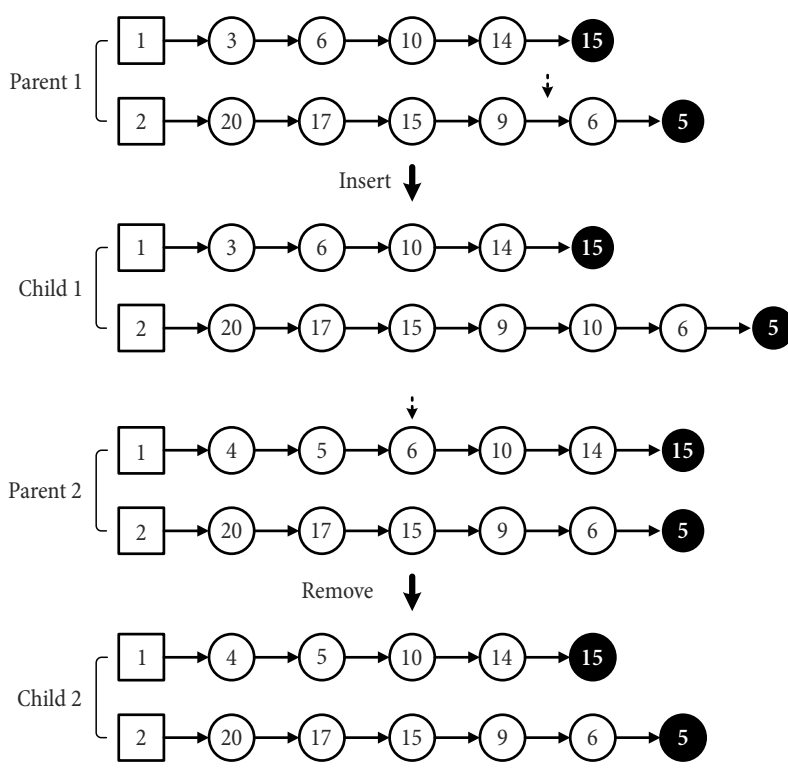

Fig. 4. Mutation operators of the GA-based approach

Table 1. Key parameters of the sample problems

\begin{tabular}{|l|c|c|c|c|c|c|c|c|}
\cline { 2 - 10 } \multicolumn{1}{c|}{} & Class 1 & Class 2 & Class 3 & Class 4 & Class 5 & Class 6 & Class 7 & Class 8 \\
\hline $\begin{array}{l}\text { Number of park-and-rides } \\
\text { (one route from each park-and-ride) }\end{array}$ & 1 & 1 & 1 & 1 & 2 & 2 & 2 & 2 \\
\hline Number of Demand nodes & 25 & 30 & 35 & 40 & 17 & 18 & 19 & 20 \\
\hline Number of problems & 20 & 20 & 20 & 20 & 20 & 20 & 20 & 20 \\
\hline
\end{tabular}


approach it can be said that the developed GA yields good results with maximum error less than $5 \%$, in a shorter time in comparison with the optimal approach.

\section{Case Study}

In this section, the developed model is used to design urban bus routes in a small city of Iran - Babol, which includes two park-and-rides and eighteen demand nodes. The city is the capital of Babol County and located in the north of Iran. The census data show that the population of Babol city was 219467 in 2012 . Urban buses and taxis are the most important public transit of Babol that serve over the $30 \%$ of the daily demand for urban transportation. In the transportation network of Babol, park-and-rides are located on the periphery of the CBD and it is aimed to design two new urban bus routes, one route from each park-and-ride. Fig. 5 shows the location of park-and-ride facilities, demand nodes and streets, in which nodes 1 and 2 indicate the location of park-and-rides and demand nodes are marked with numbers 3 to 20 .

Based on the parameters of the developed model, the input data consist of the following:

- origin-destination travel demand matrix;

- distance matrix;

- cost matrix;

- shortest path matrix.

In addition to above requirements, other parameters that used in the model are as follows: $n=20, P R=2$, $N R=2, R_{1}=R_{2}=1, B=\$ 2$ million, $L B=5 \mathrm{~km}, \theta=2$. Parameters, such as $D^{k s}$, dis $s_{i j}, c_{i j}$ and $S P^{k s}$ are obtained from origin-destination travel demand matrix, distance matrix, cost matrix and shortest path matrix respectively. For optimum solution, GAMS software was used.

a)

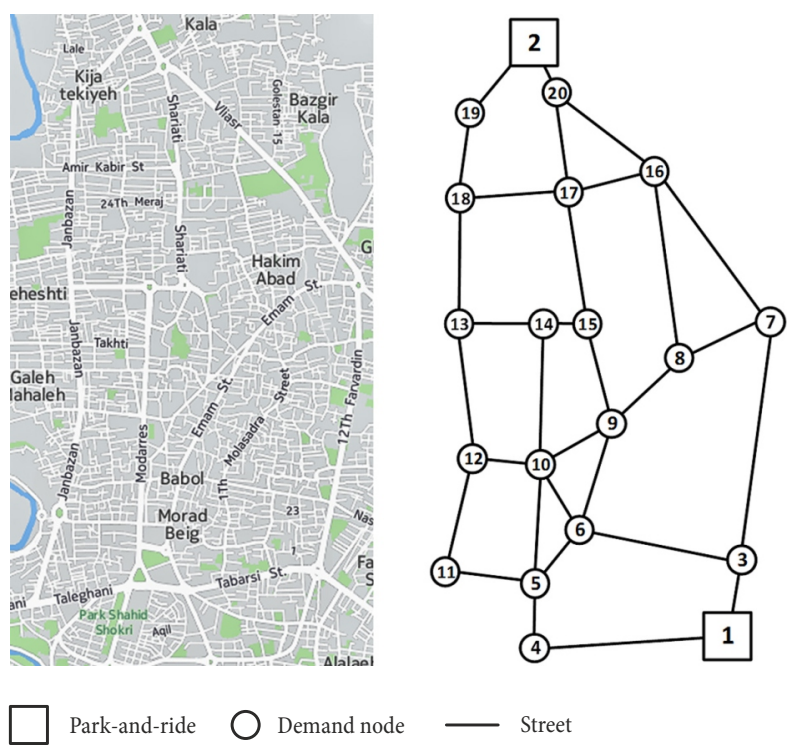

Fig. 5. Geographical map of Babol (a); transportation network of Babol (b) consisting of 2 park-and-rides and 18 demand nodes
The problem was solved under two different scenarios that each of them is described and the results of the problem under each scenario will be presented completely.

\subsection{Scenario 1}

In this scenario, it is assumed that the designer can consider all streets in designing new urban bus routes. In other words, this scenario indicates that the existing urban bus routes are not addressed in solving the problem. Therefore, constraints 16 and 17 are removed from the model formulation. After running the model under scenario 1, the urban bus routes presented in Table 2 are obtained as optimal solutions. The first route originates from park-and-ride 1 and finishes in node 9 after passing through the nodes $3,6,5,11,12$ and 10, also, parkand-ride 2 is the origin of second route and the nodes $19,18,17,16,8$ and 9 are the other nodes of this route respectively. These two routes can cover up to $19430 \mathrm{de}-$ mands, which is nearly $31 \%$ of the total demand. The length of route 1 is $5.03 \mathrm{~km}$ and the length of route 2 is $5.07 \mathrm{~km}$, also, the total cost of these two urban bus routes is $\$ 1.951$ million.

Table 2. New urban bus routes designed in scenario 1

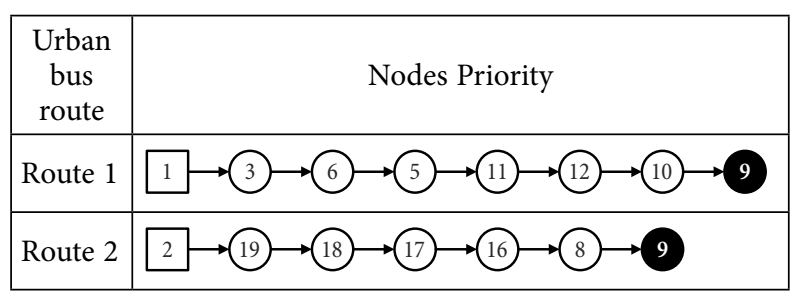

\subsection{Scenario 2}

In this scenario, the existing urban bus routes are considered in designing new urban bus routes. Hence, it can be said that the results of the problem under this scenario deal with the optimal use of existing urban bus routes. On the other hand, by adding constraints 16 and 17 to the model and taking into account the existing urban bus routes, other parameters will be as follows:

$$
\begin{aligned}
& N E=1 ; \\
& N_{1}=5 ; \\
& O_{1}=\{20,17,15,14,10\} .
\end{aligned}
$$

After solving the problem under scenario 2 and comparing the results with them of the first scenario, interesting facts were obtained which are reviewed in the following. In the second scenario, it is assumed that an urban bus route, as shown in Fig. 6, is existed on the transportation network and provides service for passengers. Use of existing urban bus route in designing new plan can lead to a decrease in the total cost and makes it possible to cover more demand than the amount given by the first scenario.

Routes obtained by solving the model under the second scenario, as shown in Table 3, are different from 


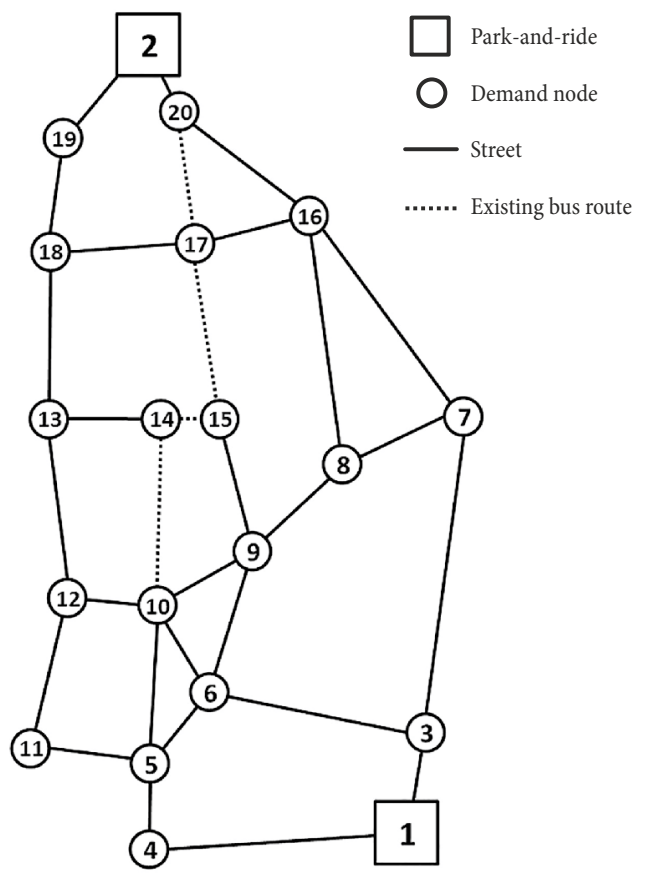

Fig. 6. The plan of existing urban bus route

them of the first scenario. The first route with $7.29 \mathrm{~km}$ length, originates from park-and-ride 1 and finishes in node 19 after passing through the nodes 3, 7, 8, 16, 17 and 18. Also, park-and-ride 2 is the origin of the second route and the nodes $20,17,15,14,10,9,6,5$ and 4 are the other nodes of this $5.82 \mathrm{~km}$ length route respectively. The total cost for setting up these two routes is $\$ 1.987$ million that is little more than the cost of the first scenario. In addition, optimal routes obtained in the second scenario can cover $41 \%$ of total demand that is equal to 25556 passengers.

\subsection{Discussion}

Above example was solved as a practical application of the proposed mathematical model that indicates the performance of the represented model in optimizing transportation network design. In this example, a transportation network consists of two park-and-rides and eighteen demand nodes has been evaluated and the developed model has been analysed under two different scenarios. Solving the model under these scenarios enables the decision maker to analyse the role of existing urban bus routes in designing new ones from different aspects, such as cost, route length and demand coverage. On the other hand, by using the parameter $\theta$, the designer can determine an appropriate value for

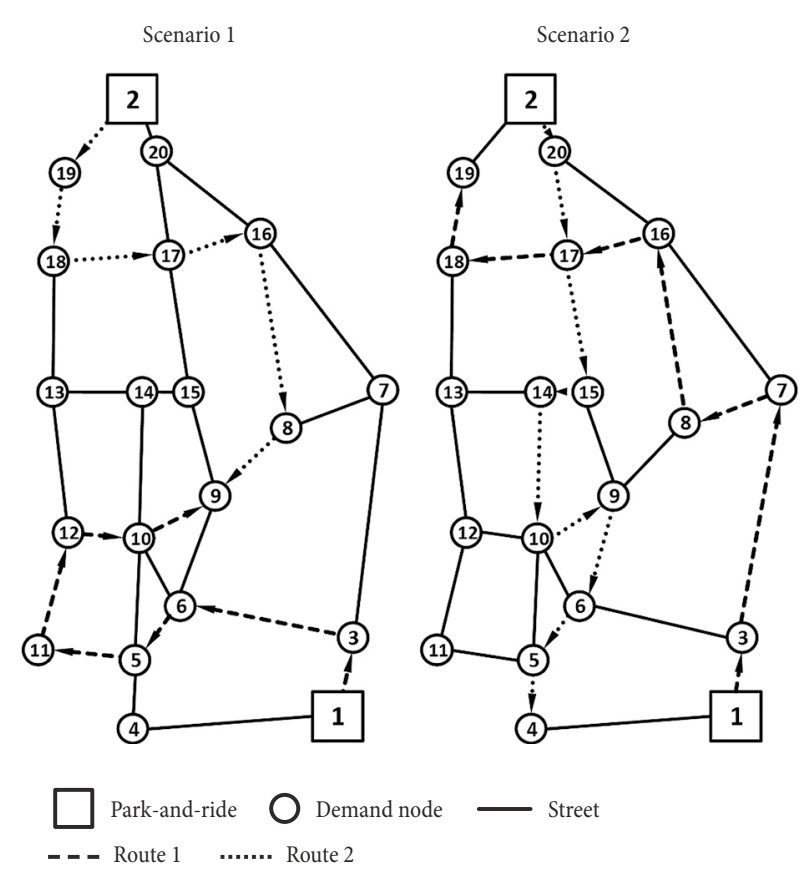

Fig. 7. Optimum urban bus routes through different scenarios

the deviation of new urban bus routes from their shortest path that is one of most important factors affecting the quality of provided service. If the designer wants to have urban bus routes with minimum deviation from the shortest path, the case $\theta=1$ gives the most direct routes as optimal solution. Minimum length of bus route is another factor, which has been represented by the parameter $L B$. The initial setting of this parameter is done according to the designers' needs and by considering characteristics of the transportation network. Value of this parameter in small, medium and large towns varies from 5 to $30 \mathrm{~km}$. In this study, according to the size of Babol and the volume of demand in its transportation network, $L B$ parameter was defined equal to $5 \mathrm{~km}$. Fig. 7 gives a graphical overview of the results of the two different scenarios which makes the comparison easier.

\section{Conclusions}

The model represented in this study helps to design urban bus routes, with park-and-ride as origin, in such a manner as to maximize the covered demand. Based on the seven factors, i.e. demand coverage, route directness, passengers' satisfaction, minimum length of bus route, budget, use of existing bus routes and number of lines, the developed model seeks to find urban bus routes in transportation network equipped with park-and-ride.

Table 3. New urban bus routes designed in scenario 2

\begin{tabular}{|c|c|}
\hline Urban bus route & Nodes Priority \\
\hline Route 1 & \\
\hline Route 2 & $\rightarrow 17 \rightarrow 15$ \\
\hline
\end{tabular}


However, none of previous studies have considered these factors simultaneously, also, the impact of park-andride location in designing new urban bus routes has not been studied before. Solving the model under the first scenario enables the designer to determine new urban bus routes without no restriction in the use of streets for designing new urban bus routes. In contrast, in the second scenario designer can consider the existing urban bus routes to design new routes. Based on obtained results, reuse of existing urban bus routes in designing new urban bus routes causes that the length of routes 1 and 2 are increased $2.26 \mathrm{~km}$ and $0.75 \mathrm{~km}$ respectively, also, it causes a $10 \%$ increase in the quantity of demand coverage.

After collecting and processing the input data, optimal solution of the model was obtained by CPLEX solver of GAMS software. The results show that, by considering 'use of existing bus routes' factor, it will be possible to invest further money in the development of new routes, which lead to an increment in their length and the covered demand. Generally, it can be said that the developed model helps the decision makers to compare the benefits and costs of different policies. Also, it uses the most common factors in designing the network of urban buses. Using the characteristics of genetic algorithm in solving urban buses network design problem can help us to find good solutions for large-scale problems, which need much time to get optimal solution. Finally, determining urban buses service frequency along with their routing, using other heuristics to solve the problem and extending the proposed methodology to design other transport network design problems are some of future research directions.

\section{References}

Amiripour, S. M. M.; Ceder, A.; Mohaymany, A. S. 2014. Hybrid method for bus network design with high seasonal demand variation, Journal of Transportation Engineering 140(6). http://doi.org/10.1061/(ASCE)TE.1943-5436.0000669

Badia, H.; Estrada, M.; Robusté, F. 2014. Competitive transit network design in cities with radial street patterns, Transportation Research Part B: Methodological 59: 161-181. http://doi.org/10.1016/j.trb.2013.11.006

Barra, A.; Carvalho, L.; Teypaz, N.; Cung, V.-D.; Balassiano, R. 2007. Solving the transit network design problem with constraint programming, in 11th World Conference on Transport Research - WCTR 2007, 24-28 June 2007, Berkeley, CA, US, 1-17.

Bielli, M.; Caramia, M.; Carotenuto, P. 2002. Genetic algorithms in bus network optimization, Transportation Research Part C: Emerging Technologies 10(1): 19-34. http://doi.org/10.1016/S0968-090X(00)00048-6

Brandão, J. 2004. A tabu search algorithm for the open vehicle routing problem, European Journal of Operational Research 157(3): 552-564.

http://doi.org/10.1016/S0377-2217(03)00238-8

Chakroborty, P.; Wivedi, T. 2002. Optimal route network design for transit systems using genetic algorithms, Engineering Optimization 34(1): 83-100.

http://doi.org/10.1080/03052150210909
Cipriani, E.; Gori, S.; Petrelli, M. 2012. Transit network design: a procedure and an application to a large urban area, Transportation Research Part C: Emerging Technologies 20(1): 3-14. http://doi.org/10.1016/j.trc.2010.09.003

Curtin, K. M.; Biba, S. 2011. The transit route arc-node service maximization problem, European Journal of Operational Research 208(1): 46-56. http://doi.org/10.1016/j.ejor.2010.07.026

Dantzig, G. B.; Harvey, R. P.; Lansdowne, Z. F.; Robinson, D. W.; Maier, S. F. 1979. Formulating and solving the network design problem by decomposition, Transportation Research Part B: Methodological 13(1): 5-17. http://doi.org/10.1016/0191-2615(79)90003-1

Fan, L.; Mumford, C. L. 2010. A metaheuristic approach to the urban transit routing problem, Journal of Heuristics 16(3): 353-372. http://doi.org/10.1007/s10732-008-9089-8

Farahani, R. Z.; Miandoabchi, E.; Szeto, W. Y.; Rashidi, H. 2013. A review of urban transportation network design problems, European Journal of Operational Research 229(2): 281-302. http://doi.org/10.1016/j.ejor.2013.01.001

Friesz, T. L. 1985. Transportation network equilibrium, design and aggregation: key developments and research opportunities, Transportation Research Part A: General 19(5-6): 413-427. http://doi.org/10.1016/0191-2607(85)90041-X

Ge, Y.-E.; Prentkovskis, O.; Tang, C.; Saleh, W.; Bell, M. G. H.; Junevičius, R. 2015. Solving traffic congestion from the demand side, Promet-Traffic\&Transportation 27(6): 529-538. http://doi.org/10.7307/ptt.v27i6.1734

Golden, B. L.; Magnanti, T. L.; Nguyen, H. Q. 1977. Implementing vehicle routing algorithms, Networks 7(2): 113148. http://doi.org/10.1002/net.3230070203

Karou, S.; Hull, A. 2014. Accessibility modelling: predicting the impact of planned transport infrastructure on accessibility patterns in Edinburgh, UK, Journal of Transport Geography 35: 1-11. http://doi.org/10.1016/j.jtrangeo.2014.01.002

Khakbaz, A.; Nookabadi, A. S.; Shetab-bushehri, S. N. 2013. A model for locating park-and-ride facilities on urban networks based on maximizing flow capture: a case study of Isfahan, Iran, Networks and Spatial Economics 13(1): 43-66. http://doi.org/10.1007/s11067-012-9172-4

Khoo, H. L.; Teoh, L. E.; Meng, Q. 2014. A bi-objective optimization approach for exclusive bus lane selection and scheduling design, Engineering Optimization 46(7): 987-1007. http://dx.doi.org/10.1080/0305215X.2013.812728

Lampkin, W.; Saalmans, P. D. 1967. The design of routes, service frequencies, and schedules for a municipal bus undertaking: a case study, Journal of the Operational Research Society 18(4): 375-397. http://dx.doi.org/10.1057/jors.1967.70

Lin, S.-W.; Yu, V. F.; Chou, S.-Y. 2010. A note on the truck and trailer routing problem, Expert Systems with Applications 37(1): 899-903. http://doi.org/10.1016/j.eswa.2009.06.077

Nikolić, M.; Teodorović, D. 2013. Transit network design by bee colony optimization, Expert Systems with Applications 40(15): 5945-5955. http://doi.org/10.1016/j.eswa.2013.05.002

Pacheco, J.; Alvarez, A.; Casado, S.; González-Velarde, J. L. 2009. A tabu search approach to an urban transport problem in northern Spain, Computers \& Operations Research 36(3): 967-979. http://doi.org/10.1016/j.cor.2007.12.002

Sariklis, D.; Powell, S. 2000. A heuristic method for the open vehicle routing problem, Journal of the Operational Research Society 51(5): 564-573.

http://doi.org/10.1057/palgrave.jors.2600924 
Szeto, W. Y.; Wu, Y. 2011. A simultaneous bus route design and frequency setting problem for Tin Shui Wai, Hong Kong, European Journal of Operational Research 209(2): 141-155. http://doi.org/10.1016/j.ejor.2010.08.020

Tay, R.; Choi, J.; Kattan, L.; Khan, A. 2011. A multinomial logit model of pedestrian-vehicle crash severity, International Journal of Sustainable Transportation 5(4): 233-249. http://doi.org/10.1080/15568318.2010.497547

Tirachini, A. 2014. The economics and engineering of bus stops: spacing, design and congestion, Transportation Research Part A: Policy and Practice 59: 37-57. http://doi.org/10.1016/j.tra.2013.10.010

Tirachini, A.; Hensher, D. A.; Rose, J. M. 2014. Multimodal pricing and optimal design of urban public transport: the interplay between traffic congestion and bus crowding, Transportation Research Part B: Methodological 61: 33-54. http://doi.org/10.1016/j.trb.2014.01.003

Thompson, K.; Schofield, P. 2007. An investigation of the relationship between public transport performance and destination satisfaction, Journal of Transport Geography 15(2): 136-144. http://doi.org/10.1016/j.jtrangeo.2006.11.004

Yan, S.; Shih, Y. L.; Wang, C. L. 2010. An ant colony systembased hybrid algorithm for square root concave cost transhipment problems, Engineering Optimization 42(11): 9831001. http://doi.org/10.1080/03052150903563751

Zhao, F. 2006. Large-scale transit network optimization by minimizing user cost and transfers, Journal of Public Transportation 9(2): 107-129. http://doi.org/10.5038/2375-0901.9.2.6

Zhao, F.; Zeng, X. 2006. Optimization of transit network layout and headway with a combined genetic algorithm and simulated annealing method, Engineering Optimization 38(6): 701-722. http://doi.org/10.1080/03052150600608917 